\title{
Contribuições de um Objeto de Aprendizagem e dos Registros de Representações Semióticas no Estudo da Função Exponencial
}

\author{
Aline Kempa Bonotto, PPGECIMAT/UNIFRA,alinnebonoto@ hotmail.com \\ Eleni Bisognin, PPGECIMAT/UNIFRA, eleni@unifra.br
}

Resumo: Este artigo descreve resultados parciais de uma investigação realizada num curso de mestrado em Ensino de Matemática, que tem como um dos propósitos analisar a contribuição de um Objeto de Aprendizagem, para o ensino e aprendizagem da função exponencial, com alunos de uma turma do Ensino Médio. A investigação é de cunho qualitativo e para fundamentar as análises dos resultados foi utilizada a teoria de registros de representação semiótica, de Duval. Os resultados mostraram que as atividades contextualizadas e a possibilidade de conversão dos diferentes registros de representação da função exponencial, propiciadas pelas atividades do Objeto de Aprendizagem, provocaram a curiosidade, o interesse, motivaram os alunos a participarem das aulas e deram significado ao conteúdo estudado.

Palavras chave: Objeto de Aprendizagem, Função Exponencial, Registros de Representação Semiótica.

\section{Contributions of a Learning Object and Semiotics Representations Registers in Exponential Function Study}

\begin{abstract}
This article describes partial results of an investigation carried out in a Master's degree in Mathematics Teaching, which has as one of the purposes of analyzing the contribution of a Learning Object to the teaching and learning of the exponential function, with students in a class of high school. The research is qualitative in nature and to support the analysis of the results we used the theory of semiotic representation registers of Duval. The results showed that the contextualized activities and the possibility of converting the different registers of representation of the exponential function, afforded by the activities of the Learning Object, provoked curiosity, interest, motivate students to participate in classes and gave meaning to study content.
\end{abstract}

Keywords: Learning Object, Exponential Function, Semiotic Representation Registers.

\section{Introdução}

Sabe-se que as Tecnologias de Informação e Comunicação (TIC), estão cada vez mais presentes no cotidiano de alunos e professores e isso tem provocado mudanças na forma como o individuo se comunica e busca informações. Tem-se observado, desde o final do século passado, mudanças profundas na sociedade devido à inserção das tecnologias da informação e isso tem afetado a escola e o processo de ensino e aprendizagem. Para atender às novas exigências da sociedade, torna-se necessário que professores utilizem, em suas aulas, recursos tecnológicos como suporte pedagógico para despertar o interesse e a motivação do aluno tornando-o mais participativo em sala de aula.

De acordo com os Parâmetros Curriculares Nacionais (PCN), a utilização de recursos computacionais na educação "possibilita a criação de ambientes de aprendizagem em que os alunos possam pesquisar, fazer antecipações e simulações, confirmar ideias prévias, experimentar, criar soluções e construir novas formas de 
representação mental" (BRASIL, 1998, p.141). Dentre os diferentes recursos computacionais destacam- se os Objetos de Aprendizagem os quais tem como propósito principal apoiar o processo de ensino e aprendizagem. De acordo com Willey (2002, p. 6), Objetos de Aprendizagem "são considerados quaisquer recursos digitais que possam ser reutilizados para suporte ao ensino" e, de acordo com Nascimento (2007), a característica principal é fazer com que "a aprendizagem se torne mais efetiva e mais profunda que a obtida pelos meios tradicionais".

Neste trabalho o Objeto de Aprendizagem é um recurso digital, reutilizável, que serve como apoio às atividades que tem por objetivo mediar os alunos na atribuição de significados aos conceitos matemáticos relativos à função exponencial.

Para enfatizar as potencialidades da informática na Educação Matemática, os Parâmetros Curriculares Nacionais (BRASIL,1998), comentam as contribuições dos recursos tecnológicos. De acordo com o documento, a tecnologia para o ensino de Matemática,

\begin{abstract}
[...] relativiza a importância do cálculo mecânico e da simples manipulação simbólica, uma vez que por meio dos instrumentos esses cálculos podem ser realizados de modo mais rápido e eficiente; evidencia para os alunos a importância do papel da linguagem gráfica e de novas formas de representação, permitindo novas estratégias de abordagem de variados problemas; possibilita o desenvolvimento, nos alunos, de um crescente interesse pela realização de projetos e atividades de investigação e exploração como parte fundamental de sua aprendizagem; permite que os alunos construam uma visão mais completa da verdadeira natureza da atividade matemática e desenvolvam atitudes positivas diante de seu estudo. (BRASIL, 1998, p. 43-44).
\end{abstract}

Cabe destacar que o desenvolvimento das Tecnologias na Educação surge como um meio de ensino e não como um fim, pois, quando usados de maneira eficiente, a utilização desses recursos tecnológicos pode modificar as formas como os alunos aprendem e são ensinados. O grande desafio dos professores não é somente utilizar tecnologias em sala de aula, mas sim, tornar a aula mais envolvente, interativa, criativa e capaz de produzir significados ao aluno por meio da utilização da tecnologia.

Para tornar motivador o ensino de Matemática, os Parâmetros Curriculares Nacionais (PCN) sugerem, também, a contextualização dos conteúdos. Esta é outra ferramenta importante para o ensino, que pode despertar o interesse dos alunos pelo fato de vincular a Matemática às atividades cotidianas.

Com a preocupação de encontrar alternativas que propiciem um ensino e aprendizagem com significado para uma turma de alunos do Ensino Médio, no estudo da função exponencial, realizou-se uma pesquisa de caráter qualitativo, tendo como um dos objetivos analisar a contribuição de um Objeto de Aprendizagem no ensino e aprendizagem da Matemática. Escolheu-se o Objeto de Aprendizagem: "Potencializando seu Conhecimento", encontrado no repositório, http://www.projetos.unijui.edu.br/matematica/fabrica_virtual/exponencial/index.html).

Esse Objeto de Aprendizagem possibilita fazer simulações e explorar as propriedades da função exponencial, além de apresentar situações contextualizadas envolvendo conceitos da Matemática Financeira. Para o desenvolvimento das atividades foi utilizada a abordagem metodológica da Investigação Matemática conforme proposta por Ponte et.al.(2009).

Nesse trabalho são apresentados resultados parciais dessa investigação que fazem parte da dissertação do curso de Mestrado em Ensino de Matemática da primeira autora. 


\title{
1-Os Objetos de Aprendizagem e os Registros de Representação Semiótica
}

Pesquisadores tais como Borba e Penteado (2002, 2003), Miskulin et al. (2006), Maltempi (2008), Tarouco et.al. (2003), Medeiros e Munhoz (2012), Menegais, Fagundes e Sauer (2014), tem destacado a importância do uso das Tecnologias da Informação e Comunicação no ensino de Matemática e ressaltado o potencial desses recursos na construção de conhecimentos. Essas tecnologias, cada vez mais presentes na vida dos professores e alunos, propiciam uma nova sistemática e dão um novo suporte ao processo de ensino e aprendizagem. Conforme destacam os Parâmetros Curriculares Nacionais (BRASIL, 1998), os recursos computacionais podem ser utilizados como fonte de informação e como um suporte para construção do conhecimento. Salientam ainda que um recurso computacional,

\begin{abstract}
pode ser um grande aliado do desenvolvimento cognitivo dos alunos, principalmente na medida em que possibilita o desenvolvimento de um trabalho que se adapta a distintos ritmos de aprendizagem e permite que o aluno aprenda com seus erros. Por outro lado, o bom uso que se possa fazer do computador na sala de aula também depende da escolha de softwares, em função dos objetivos que se pretende atingir e da concepção de conhecimento e de aprendizagem que orienta o processo. (BRASIL, 1998, p. 44).
\end{abstract}

Entre as diferentes tecnologias que dão suporte ao ensino destacam-se os Objetos de Aprendizagem.

Existem diferentes conceitos sobre Objeto de Aprendizagem. Para Willey (2002, p. 6), um Objeto de Aprendizagem é "qualquer recurso digital que possa ser reutilizados para suporte ao ensino". Sobre esse conceito, Dantas e Julio (2014, p.446), exemplificam as três características principais de um Objeto de Aprendizagem: como recurso digital, os autores dizem que Objetos de Aprendizagem "podem ser considerados desde simples apresentação de slides a jogos implementados por computação gráfica". Sobre a característica da reutilização os autores salientam que é a "possibilidade de ser utilizado em diferentes contextos bastando para isso a inserção de novos valores de entrada" e o suporte ao ensino "consiste em sua utilização didática, como algo que tenha relação com o conteúdo em estudo e que possibilite a produção de significados de acordo com os objetivos da aula". (DANTAS, JULIO, 2014, p.446).

O fato de os Objetos de Aprendizagem serem apresentados, na sua maioria, por meio de recursos computacionais e apresentar situações ligadas a realidade, eles são motivadores e despertam o interesse do aluno. Porém, para que esses Objetos sejam recursos tecnológicos eficientes para o processo de ensino, torna-se necessário que o professor conheça suas potencialidades e limitações e que saiba relacionar os objetivos de ensino e os conceitos que podem ser explorados. Para seu uso o professor deve levar em consideração as necessidades e interesses dos alunos e o nível de interatividade que o Objeto proporciona. Para serem eficazes eles devem propiciar a realização de atividades que sejam desafiadoras para que o aluno possa explorar o conteúdo e se sentir desafiado.

Quanto a utilização de recursos tecnológicos no ensino, resultados de uma pesquisa realizada com o propósito de investigar o impacto da inserção de tecnologias digitais na formação inicial de professores de Matemática, em egressos de uma universidade pública federal, as pesquisadoras Menegais, Fagundes e Sauer (2014) concluiram que "as disciplinas voltadas ao uso pedagógico das tecnologias, em particular, a disciplina de Tecnologias Aplicadas ao Ensino de Matemática, foram muito importantes para a formação inicial dos professores, mas não se mostraram suficientes para o uso efetivo nas práticas em sala de aula".(p.1). 
Para essa pesquisa foi selecionado o Objeto de Aprendizagem "Potencializando seu Conhecimento" que aborda a função exponencial, apresenta problemas contextualizados envolvendo a Matemática Financeira, inclui um propósito de aprendizagem e avaliação e oportuniza textos, gráficos, figuras e simulações.

As simulações e os gráficos, propiciados pela exploração das atividades nesse Objeto de Aprendizagem, favorecem a utilização de diferentes representações para o estudo da função exponencial. Nesse sentido, para análise dos resultados, fez-se uso da Teoria dos Registros de Representação Semiótica de Duval.

De acordo com Duval (2003), o desenvolvimento das representações semióticas é uma condição essencial para a evolução do pensamento matemático. Para o autor representações semióticas podem ser um enunciado em língua natural, um gráfico, uma tabela, uma fórmula, etc. Elas servem para que um indivíduo expresse, por meio delas, suas representações mentais e, essas representações são essenciais à atividade cognitiva do pensamento e à produção de conhecimentos.

Conforme afirma Duval (2012), a articulação entre diferentes registros de representação como, por exemplo, sistemas de escrita (numérica, algébrica ou simbólica), figuras geométricas, gráficos, é condição necessária para a compreensão em Matemática. $\mathrm{O}$ autor afirma que,

[...] é essencial, na atividade matemática, poder mobilizar muitos registros de representação semiótica (figuras, gráficos, escrituras simbólicas, língua natural, etc...) no decorrer de um mesmo passo, poder escolher um registro no lugar de outro. E, independente de toda comodidade de tratamento, o recurso a muitos registros parece mesmo uma condição necessária para que os objetos matemáticos não sejam confundidos com suas representações e que possam também ser reconhecidos em cada uma de suas representações. (DUVAL, 2012, p. 270).

É importante o professor, ao planejar sua aula, utilizar mais de um registro de representação e estabelecer uma coordenação entre eles. A coordenação, de acordo com Duval (2012, p.292), se manifesta "pela rapidez e espontaneidade da atividade cognitiva da conversão." A conversão diz respeito às transformações das representações. Duval (2009, p.39), se refere às transformações como sendo o tratamento e a conversão. O autor salienta que o "tratamento se efetua no interior de um mesmo registro, aquele onde as regras de funcionamento são utilizadas" e, apenas um registro de representação é mobilizado. A conversão é a transformação que permite a passagem de um registro a outro. (DUVAL, 2009, p.39).

\section{2-Procedimentos Metodológicos}

A abordagem metodológica empregada nessa pesquisa é de cunho qualitativo. Segundo Ludke e André (1986), "a pesquisa qualitativa tem o ambiente natural como sua fonte direta de dados e o pesquisador como seu principal instrumento." (p. 11). A pesquisa qualitativa é essencialmente descritiva, pois ela enfatiza a descrição dos fenômenos e os significados dados pelos participantes.

Participaram da pesquisa vinte alunos do Ensino Médio de uma escola pública em que a professora atua como docente e as aulas foram desenvolvidas no laboratório de informática da escola. Foi solicitado que as atividades fossem trabalhadas em duplas, na qual a escolha ficou a critério dos próprios alunos. Foi escolhido o Objeto de Aprendizagem "Potencializando seu Conhecimento", para o estudo da função exponencial. 
A professora pesquisadora teve participação direta na pesquisa e os dados foram coletados no ambiente natural. Como instrumentos de coleta de dados foram utilizados a observação participante, o diário de campo da professora e os registros feitos pelos alunos.

Conforme Becker (1994, p. 47) na observação participante,

\begin{abstract}
O observador participante coleta dados através da sua participação na vida cotidiana do grupo organizado que estuda. Ele observa as pessoas que esta estudando para ver as situações com que se deparam normalmente e como se comportam diante delas. Entabula conversação com alguns ou com todos os participantes desta situação e descobre as interpretações que eles têm sobre os acontecimentos que observou. (BECKER, 1994, p. 47).
\end{abstract}

Sobre o diário de campo Fiorentini e Lorenzato (2006), colocam que o diário de campo é onde "o pesquisador registra observações de fenômenos, faz descrições de pessoas e cenários, descreve episódios ou retrata diálogos". (FIORENTINI; LORENZATO, 2006, p. 119).

Como a pesquisa foi realizada no laboratório de informática e para que os registros fossem os mais fidedignos possíveis, a professora pesquisadora registrou as observações em seu diário ao término das aulas.

Para obtenção dos dados, além do diário de campo da pesquisadora, foram recolhidas as produções dos alunos, para serem e extraídas informações quanto à forma como eles resolveram os problemas propostos.

Para análise dos dados foram considerados as estratégias e conhecimentos utilizados pelos alunos no desenvolvimento das atividades propostas, suas percepções e considerações em relação ao tema e as atividades propostas no Objeto de Aprendizagem.

Buscou-se encontrar nas atuais tendências metodológicas de ensino da Matemática, referências para elaborar uma proposta visando tornar o ensino de função exponencial mais significativo e compreensível aos alunos. O propósito é fazer com que o aluno realmente participe do processo de construção do seu conhecimento, tendo a oportunidade de refletir, indagar, discutir, formular hipóteses e expor suas idéias em relação ao objeto de estudo. Optou-se pela perspectiva metodológica da Investigação Matemática, conforme Ponte et al.(2009).

A escolha por essa metodologia deve-se ao fato dela propiciar ao aluno a oportunidade de investigar e explorar diferentes formas para solucionar as atividades propostas no Objeto de Aprendizagem, além de possibilitar a formulação de conjecturas e verificação de sua veracidade pois, o Objeto de Aprendizagem, permite fazer simulações.

O encaminhamento das atividades investigativas seguiu os passos segundo Ponte, Brocardo e Oliveira (2009). Segundo esses autores uma atividade investigativa no contexto de sala de aula desenvolve-se em três fases: introdução da tarefa, desenvolvimento da atividade investigativa e discussão da tarefa.

A primeira fase que é a introdução da tarefa é uma fase importante pois, ela direciona o encaminhamento da investigação. A professora introduziu as tarefas deixando claro o objetivo e o sentido de cada atividade.

$\mathrm{Na}$ segunda fase que é o desenvolvimento da atividade investigativa, os alunos fizeram explorações, testes e formularam conjecturas para resolverem as questões propostas. Essa é uma fase que é considerada por Ponte, Brocardo e Oliveira (2009), o caminho mais longo. É uma fase que demanda um tempo maior, pois é onde os alunos realizam explorações e formulam conjecturas, é uma fase onde eles conseguem compreender o sentido da atividade proposta. 
A última fase, a discussão da tarefa foi um dos momentos mais significantes da investigação, pois os alunos compartilharam resultados e sistematizaram suas principais idéias com toda a turma.

\section{3-Análise dos Resultados}

O Objeto de Aprendizagem "Potencializando seu Conhecimento" utilizado nesta investigação apresenta situações problema, por meio de atividades interativas, que exploram a realidade do dia-a-dia dos alunos. A primeira atividade aborda a compra de aparelhos celulares, computadores e televisores, bem como explora fatores relacionados ao tipo de compra realizada; ou seja, se a compra for a prazo existirão várias formas de pagamento.

Inicialmente ao acessar o Objeto de Aprendizagem o aluno é convidado a entrar na loja "Meu sonho de Consumo" e efetuar a compra de um dos itens disponíveis.

O produto escolhido pela dupla D3 foi um computador e os alunos tinham duas opções de escolha, podiam selecionar o Modelo 1 ou o Modelo 2 para finalizar a compra.

Após as escolhas dos itens os alunos podem perguntar ao vendedor quais são as condições de parcelamento que a loja oferece. Então, o vendedor sugere ao cliente que, antes de definir sua escolha de pagamento, ele conheça um pouco mais sobre o tema juro composto, para assim poder fazer a melhor escolha.

Nas telas seguintes o Objeto de Aprendizagem apresenta uma situação-problema com uma tabela que deve ser preenchida pelos alunos de acordo com os dados fornecidos, indicando o valor de compra, a taxa de juros cobrada pela loja e o valor da aplicação em caderneta de poupança para oportunizar aos alunos fazerem um comparativo. Essa atividade permitiu fazer simulações sobre as vantagens, ou desvantagens, da compra a prazo. Para cada dupla os valores foram distintos.

A abordagem metodológica da Investigação Matemática foi adequada na busca de solução dessa atividade, pois, as simulações propiciadas pelo Objeto de Aprendizagem, permitiram à professora fazer perguntas e, aos alunos, formularem e verificarem suas conjecturas. Uma das perguntas feita pela professora foi: é possível obter-se uma expressão para o cálculo do montante ao final de t períodos de tempo?

Cada dupla tentou explicar como tinha obtido o resultado e, na socialização dos resultados, pode-se construir a função $M=C(1+i)^{t} \quad$ em que $M$ representa o montante, $C$ o capital, $i$ a taxa e a variável $t$ o tempo.

Na figura 1 é mostrado o processo de obtenção da função que permite calcular o montante. 
Figura 1: Tela do Objeto de Aprendizagem

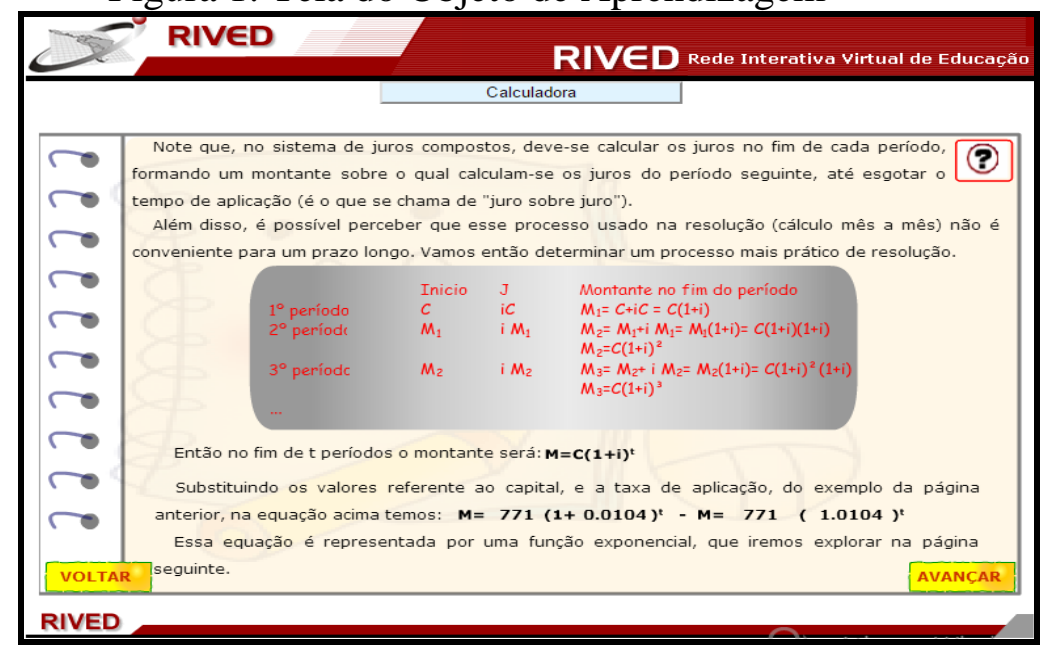

Fonte: RIVED OA - Potencializando seu conhecimento

A descrição do processo favoreceu a construção do conceito de função exponencial e buscar uma generalização. Definiu-se a função exponencial como sendo a função definida por $f(x)=a^{x}$ para x assumindo valores reais e $a \neq 0, a>1$ ou $0<a<1$. Essa é uma representação algébrica da função exponencial.

Nas próximas atividades são exploradas as propriedades da função exponencial e seu gráfico. Para isso foram feitas simulações variando o valor da base " $a$ ". Para efetuar as simulações basta o aluno clicar nas setas mostradas na tela do Objeto de Aprendizagem da figura 2.

Figura 2: Tela do Objeto de Aprendizagem

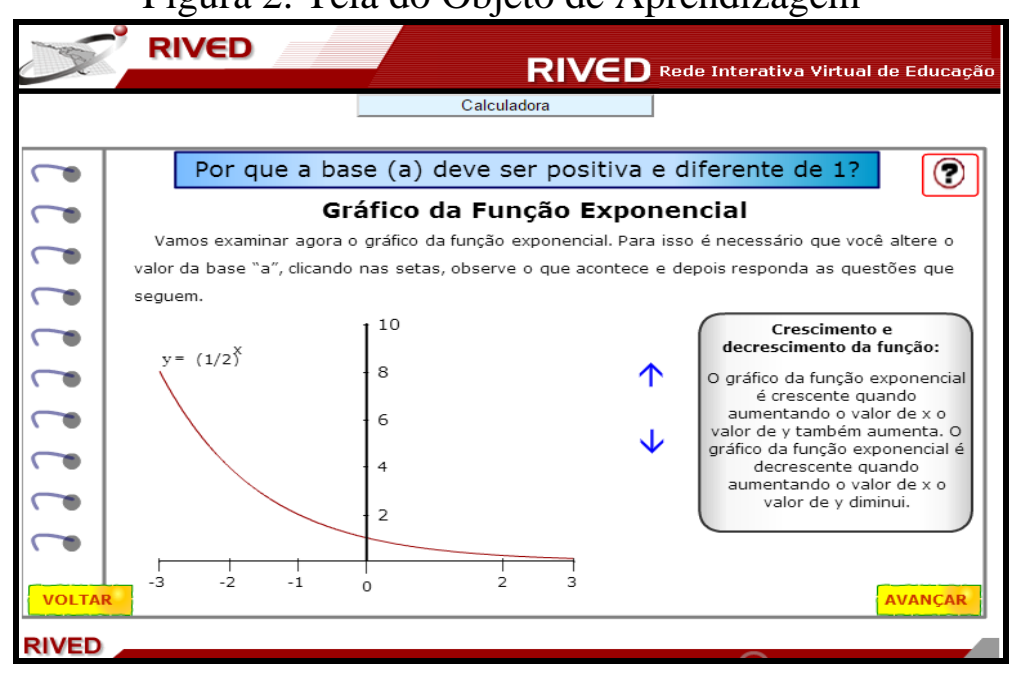

Fonte: RIVED OA - Potencializando seu conhecimento

Os alunos efetuaram várias simulações, variando o valor da base $a$, para concluírem o comportamento do gráfico da função e completarem a atividade proposta como mostrado na figura 3 , a seguir.

Figura 3: Tela do Objeto de Aprendizagem 


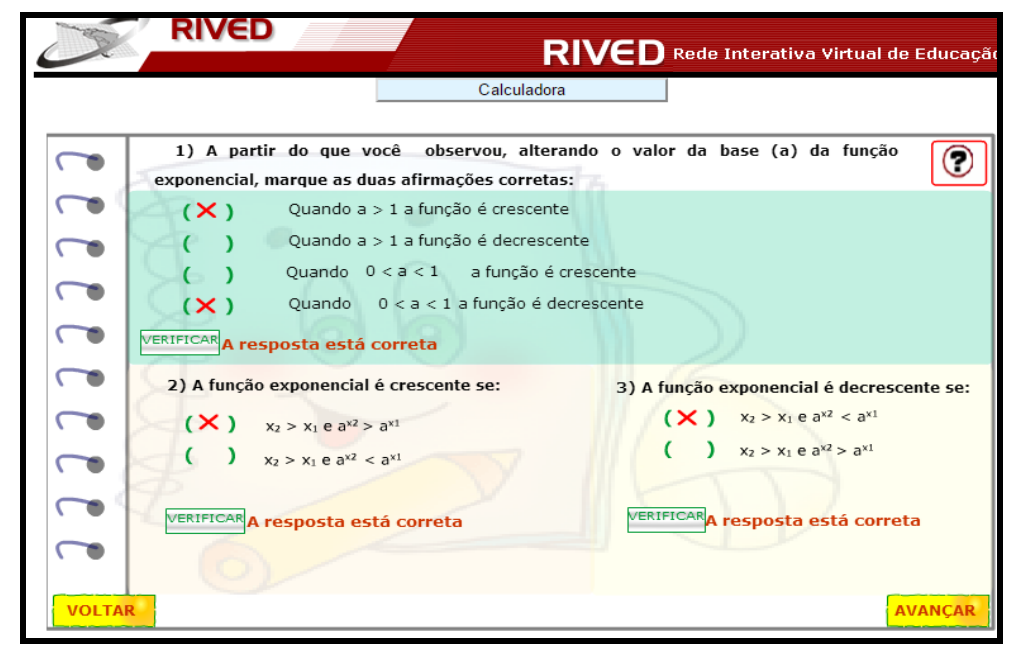

Fonte: RIVED OA - Potencializando seu conhecimento

Os alunos utilizaram, também, os recursos do software Geogebra para fazerem as simulações e analisarem o comportamento gráfico da função exponencial $f(x)=$ $b(a)^{c x}$, quando os parâmetros reais $a, b e c$ variam.

A professora colocou algumas questões para auxiliar os alunos a chegarem a uma conclusão, como por exemplo: o que acontece ao gráfico de $f(x)=2^{x}$ quando multiplica-se a função por uma constante c $>0$ ? Ou uma constante c $<0$ ?

As imagens produzidas pela dupla $\mathrm{D}_{1}$ para diferentes valores de $c$, são mostradas na figura 4 , a seguir.

Figura 4: Representações gráficas feitas no Geogebra pela dupla D1

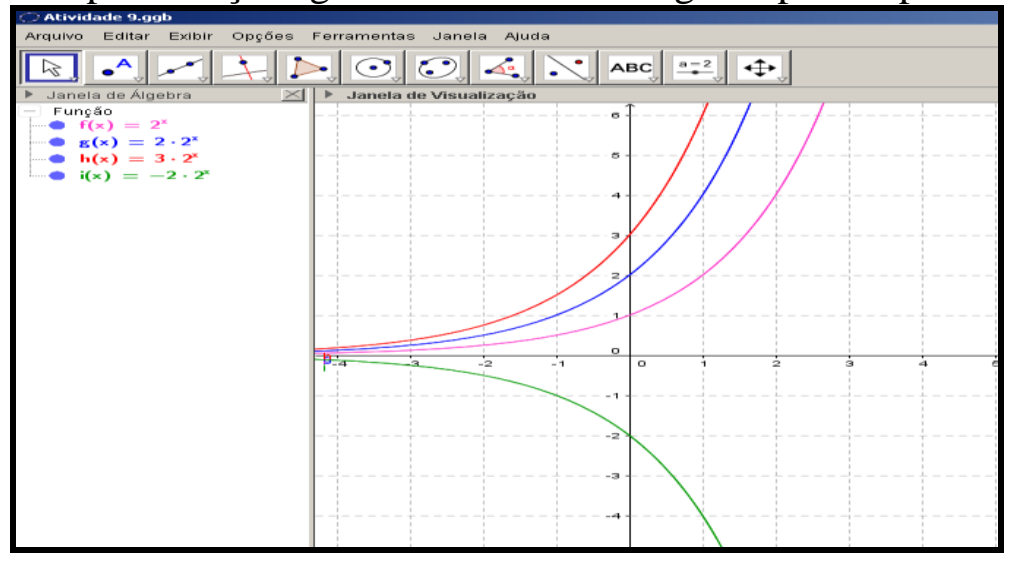

Fonte: Dados da pesquisa

Questões semelhantes foram colocadas pela professora considerando a base $0<\mathrm{a}<1$ e diferentes valores para os parâmetros $b$ e $c$ e quando se soma uma constante à variável $x$ ou à função.

A partir de exemplos particulares os alunos generalizaram as propriedades e expressaram essa generalização de forma gráfica e algébrica. As simulações oportunizaram a construção dos diferentes gráficos e propiciaram a comparação e a conversão entre esses registros algébricos e gráficos.

Duval (2003), coloca que, 
Há por trás da aplicação de uma regra de decodificação para passar de uma equação a um gráfico cartesiano, a necessária articulação entre as variáveis cognitivas que são específicas do funcionamento de cada um dos dois registros. Pois são essas variáveis que permitem determinar quais as unidades de significado pertinentes, que devem ser levadas em consideração, em cada um dos dois registros. (DUVAL, 2003, p.17).

Justifica-se desse modo a importância da utilização de mais de uma forma de tratamento para a conversão das representações, pois, é a conversão que facilita a compreensão e a construção do conhecimento.

\section{Considerações Finais}

O uso de um Objeto de Aprendizagem na construção de conceitos matemáticos foi utilizado como uma alternativa para tentar superar as dificuldades de compreensão e de interesse dos alunos. O Objeto "Potencializando o seu conhecimento", apresentado nesse trabalho, inseriu os alunos em situações do mundo real, nas quais foi possível a construção do conceito e significado da função exponencial para alunos do Ensino Médio, pois, nas primeiras atividades os alunos foram desafiados a escolher a melhor opção de compra considerando as taxas de juros cobradas na compra a prazo e o lucro obtido numa aplicação financeira. Durante a socialização das estratégias de resolução, foi possível estabelecer uma discussão sobre as opções que são oferecidas no comércio e como utilizar os conceitos de Matemática Financeira, para fazer a melhor escolha.

Por meio das simulações apresentadas no Objeto foi oportunizado ao aluno reconhecer, interpretar e descrever o comportamento gráfico da função provocado pela mudança de parâmetros. Entretanto, percebeu-se que o desenvolvimento das atividades do Objeto de Aprendizagem por si só, não garantiu a aquisição dos conceitos referente à função exponencial, pois os alunos conseguem "avançar" nas atividades do Objeto, realizando tentativas sucessivas de acerto e erro, sem justificar. Assim, é necessária uma intervenção do professor promovendo um ambiente de discussão e análise das estratégias dos alunos, das conjecturas levantadas para que as mesmas sejam validadas ou reformuladas. Nesse sentido, a abordagem metodológica da Investigação Matemática contribuiu para o levantamento e comprovação ou refutação das conjecturas, feitas pelos alunos, o que facilitou a compreensão do conceito de função exponencial.

Duval (2009), coloca que as diferentes representações semióticas também são necessárias à conceitualização e, para que o aluno tenha compreensão dos conceitos matemáticos, é necessário que ele tenha a capacidade de efetuar a mudança de registros e saber articulá-los naturalmente.

Ao concluir, pode-se afirmar que o Objeto de Aprendizagem "Potencializando seu conhecimento" foi um recurso que facilitou a construção do conceito de função exponencial, propiciou ao aluno fazer generalizações, conjecturar, testar hipóteses por meio da exploração das situações apresentadas, favoreceu a interatividade e despertou o interesse e participação em sala de aula.

\section{Referências}

BECKER, H. S. Métodos de pesquisa em ciências sociais. 2. ed. São Paulo: Hucitec, 1994.

BORBA, M. C.; PENTEADO, M. G. Informática e Educação Matemática. Belo Horizonte: Autêntica, 2003. 
Pesquisa em Informática e Educação Matemática. Educação em Revista, n.36, p. 239-253, Belo Horizonte, UFMG, dez. 2002.

BRASIL, Ministério da Educação e dos Desportos. Parâmetros Curriculares Nacionais: Matemática. Brasília SEF, 1998.

BRASIL, Ministério da Educação. Secretaria de Educação a Distância. Objetos de Aprendizagem: uma proposta de recurso pedagógico. Brasília: MEC, SEED, 2007.

DANTAS, S.C.; JULIO, R.S. O uso de objetos de aprendizagem em um processo de produção de significados sobre trigonometria. Acta Scientiae, v.16, n.3, Canoas: Ed. ULBRA, 2014.

DUVAL, R.; Registros de representação semiótica e funcionamento cognitivo da compreensão em matemática. In: MACHADO, S.D.A. (Org.). Aprendizagem em matemática: registros de representação semiótica. Campinas: Papirus, 2003, p.11-33.

DUVAL, R. Semiósis e pensamento humano: registros semióticos e aprendizagens intelectuais. São Paulo: Editora Livraria da Física, 2009.

DUVAL, R. Registros de representação semiótica e funcionamento cognitivo do pensamento. REVEMAT, Florianópolis, v. 7, n. 2, 2012.

LÜDKE, M; ANDRÉ, M. E. D. A. Pesquisa em educação: abordagens qualitativas. São Paulo: EPU, 1986.

MALTEMPI, M. V. Educação Matemática e Tecnologias Digitais: reflexões sobre prática e formação docente. Acta Scientiae, v.10, n.1, jan./jun., Canoas: Ed. ULBRA, 2008.

MEDEIROS, L. F.; MUNHOZ, A. S. Materiais didáticos como objetos de aprendizagem: inovação pedagógica ou solução econômica? RENOTE - Revista Novas Tecnologias na Educação, Porto Alegre, v. 10, n. 3, dez. 2012.

MENEGAIS, D. A. F. N.; FAGUNDES, L. da C.; SAUER, L.Z. Impacto da Inserção de Tecnologias Digitais na Formação Inicial de Professores de Matemática Egressos de uma Universidade Pública Federal In: RENOTE - Revista Novas Tecnologias na Educação, Porto Alegre, v. 12, n. 2, 2014

MISKULIN, S. G. R. et al. Identificação e análise das dimensões que permeiam a utilização das Tecnologias de Informação e Comunicação nas aulas de Matemática no contexto da formação de professores. Bolema, Rio Claro: UNESP, v.19, n.26, 2006.

PONTE, J. P.; BROCADO, J.; OLIVEIRA, H. Investigações Matemáticas na sala de aula. Belo Horizonte: Autêntica, 2009.

TAROUCO, L. M. R.; FABRE, M. J. M.; TAMUSIUNAS, F. R. Reusabilidade de objetos educacionais. RENOTE - Revista Novas Tecnologias na Educação. Porto Alegre, v. 1 n. 1, fev. 2003. 
WILLEY, D. A. Connecting learning objects to instructional design theory: A definition, a metaphor, and a taxionomy. 2002. Disponivel em http://reusability.org/read/chapters/wiley.doc. Acesso em 05 Abr.2015. 